\title{
THE RADIUS OF CONVEXITY OF CERTAIN ANALYTIC FUNCTIONS II
}

\author{
J.S. RATTI \\ Department of Mathematics \\ University of South Florida \\ Tampa, Florida 33620 \\ (Received August 7, 1979)
}

ABSTRACT. In [2], MacGregor found the radius of convexity of the functions $f(z)=z+a_{2} z^{2}+a_{3} z^{3}+\ldots$, analytic and univalent such that $\left|f^{\prime}(z)-1\right|<1$. This paper generalized MacGregor's theorem, by considering another univalent function $g(z)=z+b_{2} z^{2}+b_{3} z^{3}+\ldots$ such that $\left|\frac{f^{\prime}(z)}{g^{\prime}(z)}-1\right|<1$ for $|z|<1$. Several theorems are proved with sharp results for the radius of convexity of the subfamilies of functions associated with the cases: $g(z)$ is starlike for $|z|<1, g(z)$ is convex for $|z|<1, \operatorname{Re}\left\{g^{\prime}(z)\right\}>\alpha(\alpha=0,1 / 2)$.

KEY WORDS AND PHRASES. Univalent, analytic, starlike, convex, radius of starlikeness and radius of convexity.

1980 MATHEMATICS SUBJECT CLASSIFICATION CODES. 30A32, 30A36, $30 A 42$.

1. INTROUDCTION.

Throughout we suppose that $f(z)=z+a{ }_{2} z^{2}+\ldots$ is analytic for $|z|<$ and $g(z)=z+b_{2} z^{2}+\ldots$ is analytic and univalent for $|z|<1$. In [4] the author solved the following problem: what is the radius of convexity of the family of 
functions $f(z)$ which satisfy $\operatorname{Re}\left(\frac{f^{\prime}(z)}{g^{\prime}(z)}\right)>0$ for $|z|<1$ ? The problem was solved also for each of the subfamilies associated with the cases: $g(z)$ is starlike for $|z|<1, \operatorname{Re}\left\{g^{\prime}(z)\right\}>\alpha\left(\alpha=0, \frac{1}{2}\right)$ for $|z|<1, g(z)$ is convex of order $\alpha(0 \leq \alpha<1)$ for $|z|<1$.

In this paper we consider functions $f(z)$ which satisfy $\left|\frac{f^{\prime}(z)}{g^{\prime}(z)}-1\right|<1$ for $|z|<1$. The radius of convexity of this family of functions is determined. Also, we find the radius of convexity for the subfamilies associated with each of the cases: $g(z)$ is starlike for $|z|<1, g(z)$ is convex for $|z|<1, \operatorname{Re}\left\{g^{\prime}(z)\right\}>\alpha\left(\alpha=0, \frac{1}{2}\right)$. The case $g(z)=z$ has already been proved by MacGregor $[2]$.

2. The following lemmas will be used in the proofs of our theorems.

LEMMA 1. [4] The function $h(z)$ is analytic for $|z|<1$ and satisfies $h(0)=1$ and $\operatorname{Re}\{h(z)\}>\alpha \quad(0 \leq \alpha<1)$ for $|z|<1$ if and only if $h(z)=\frac{1+(2 \alpha-1) z \phi(z)}{1+z \phi(z)}$, where $\phi(z)$ is analytic and satisfies $|\phi(z)| \leq 1$ for $|z|<1$.

LEMMA 2. If $\phi(z)$ is analytic for $|z|<1$ and $|\phi(z)| \leq 1$ for $|z|<1$, then

$$
\begin{aligned}
& \text { (i) }\left|\phi^{\prime}(z)\right| \leq \frac{1-|\phi(z)|^{2}}{1-|z|^{2}} \\
& \text { (ii) }\left|\frac{z \phi^{\prime}(z)+\phi(z)}{1+z \phi(z)}\right| \leq \frac{1}{1-|z|}
\end{aligned}
$$

Part (i) of Lemma 2 is well-known $[3]$, and part (ii) follows easily, by first applying triangular inequalities and then using part (i).

LEMMA 3. If $h(z)=1+c_{1} z+\ldots$ is analytic for $|z|<1$ and $\operatorname{Re}\{h(z)\}>0$ for $|z|<1$, then

$$
\operatorname{Re}\{h(z)\} \geq \frac{1-|z|}{1+|z|}
$$

This is a well-known result due to $C$. Carathéodory.

3. THEOREM 1. Suppose $f(z)=z+a_{2} z^{2}+\ldots$ is analytic for $|z|<1$ and $g(z)=z+b_{2} z^{2}+\ldots$ is analytic and univalent for $|z|<1$. 
If $\left|\frac{f^{\prime}(z)}{g^{\prime}(z)}-1\right|<1$ for $|z|<1$, then $f(z)$ maps $|z|<1 / 5$ onto a convex domain. The result is sharp.

PROOF. Let $h(z)=\frac{f^{\prime}(z)}{g^{\prime}(z)}-1$. The function $g(z)$ is univalent for $|z|<1$, therefore $g^{\prime}(z) \neq 0$ for $|z|<1$. The function $h(z)$ is analytic for $|z|<1$, $h(0)=0$ and $|h(z)|<1$ for $|z|<1$. Thus by Schwarz's lemma we have

$$
h(z)=z \phi(z) \text { with }|\phi(z)| \leq 1 \text {. }
$$

Therefore

$$
f^{\prime}(z)=g^{\prime}(z)(1+z \phi(z))
$$

Taking the logarithmic derivative we obtain

$$
\frac{f^{\prime \prime}(z)}{f^{\prime}(z)}=\frac{g^{\prime \prime}(z)}{g^{\prime}(z)}+\frac{z \phi^{\prime}(z)+\phi(z)}{1+z \phi(z)}
$$

Using lemma 2 (ii) we get

$$
\operatorname{Re}\left\{\frac{z f^{\prime \prime}(z)}{f^{\prime}(z)}+1\right\} \geq \operatorname{Re}\left\{\frac{z g^{\prime \prime}(z)}{g^{\prime}(z)}+1\right\}-\frac{|z|}{1-|z|} \cdot
$$

Since $g(z)$ is univalent, we have $[1]$

$$
\operatorname{Re}\left\{\frac{z g^{\prime \prime}(z)}{g^{\prime}(z)}+1\right\} \geq 1+\frac{|z|(2|z|-4)}{1-|z|^{2}} \text {. }
$$

Using this estimate in (3.1) we obtain

$$
\left.\operatorname{Re} \frac{z f^{\prime \prime}(z)}{f^{\prime}(z)}+1\right\} \geq \frac{1-5|z|}{1+|z|^{2}} \text {. }
$$

This last expression is positive if $|z|<1 / 5$. Since the condition $\operatorname{Re}\left\{\frac{z f^{\prime \prime}(z)}{f^{\prime}(z)}+1\right\}$ $>0$ for $|z|<r$ is necessary and sufficient for $f(z)$ to map $|z|<r$ onto a convex domain, we conclude that $f(z)$ maps $|z|<1 / 5$ onto a convex domain. To show that the estimate obtained in the theorem is sharp, we consider the function $f(z)$ such that $f^{\prime}(z)=\frac{(1+z)^{2}}{(1-z)^{3}}$ with $g(z)=\frac{z}{(1-z)^{2}}$.

This function $f(z)$ satisfies the hypotheses of the theorem and a short computation shows that $\frac{z f^{\prime \prime}(z)}{f^{\prime}(z)}+1=\frac{1+5 z}{1-z^{2}}$. This expression vanishes at $z=-1 / 5$. THEOREM 2. Let $f(z)=z+a_{2} z^{2}+\ldots$ be analytic for $|z|<1$ and 
$g(z)=z+b z^{2}+\ldots$ be analytic and starlike for $|z|<1$. If $\left|\frac{f^{\prime}(z)}{g^{\prime}(z)}-1\right|<1$ for $|z|<1$, then $f(z)$ maps $|z|<1 / 5$ onto a convex domain. The result is sharp. PROOF: Since $g(z)$ is starlike for $|z|<1$ implies $g(z)$ is univalent there, the proof of this theorem follows from that of theorem 1 .

THEOREM 3. Suppose $f(z)=z+a_{2} z^{2}+\ldots$ is analytic for $|z|<1$ and $g(z)=z+b_{2} z^{2}+\ldots$ is analytic and convex for $|z|<1$. If $\left|\frac{f^{\prime}(z)}{g^{\prime}(z)}-1\right|<1$ for $|z|<1$, then $f(z)$ maps $|z|<1 / 3$ onto a convex domain. The result is sharp. PROOF. Since $g(z)$ is convex for $|z|<1$ it is univalent there. Therefore $g^{\prime}(z) \neq 0$ for $|z|<1$ and $\operatorname{Re}\left\{\frac{z g^{\prime \prime}(z)}{g^{\prime}(z)}+1\right\}>0$ for $|z|<1$.

The function $\frac{z^{\prime \prime}(z)}{g^{\prime}(z)}+1=1+c_{1} z+\ldots$ is regular for $|z|<1$ and has positive real part, therefore by lemma 3 ,

$$
\operatorname{Re}\left\{\frac{z g^{\prime \prime}(z)}{g^{\prime}(z)}+1\right\} \geq \frac{1-|z|}{1+|z|} \text {. }
$$

Using this estimate in (3.1) we get

$$
\left.\operatorname{Re}\left\{\frac{z f^{\prime \prime}(z)}{f^{\prime}(z)}+1\right\} \geq \frac{1-|z|}{1+|z|}-\frac{|z|}{1-|z|}=\frac{1-3|z|}{1-|z|^{2}}\right) .
$$

This last expression is positive for $|z|<1 / 3$. Thus $f(z)$ maps $|z|<1 / 3$ onto a convex domain. To see that the estimate obtained is sharp, we consider $f(z)$ such that $f^{\prime}(z)=\frac{1+z}{(1-z)^{2}}$, with $g(z)=\frac{z}{1-z}$. Thus $f(z)$ satisfies the hypotheses of the theorem. However $\frac{z f^{\prime \prime}(z)}{f^{\prime}(z)}+1=\frac{1+3 z}{1-z^{2}}$, which vanishes at $z=-1 / 3$.

THEOREM 4. Suppose $f(z)=z+a_{2} z^{2}+\ldots$ is analytic for $|z|<1$ and $g(z)=z+b_{2} z^{2}+\ldots$ is analytic and $\operatorname{Re} g^{\prime}(z)>0$ for $|z|<1$. If $\left|\frac{f^{\prime}(z)}{g^{\prime}(z)}-1\right|<1$ for $|z|<1$, then $f(z)$ maps $|z|<(\sqrt{17}-3) / 4$ onto a convex domain. The result is sharp.

PROOF. Since $\operatorname{Re} g^{\prime}(z)>0$ for $|z|<1$, it follows from lemma 1 , with $\alpha=0$ 
that

$$
g^{\prime}(z)=\frac{1-z \phi(z)}{1+z \phi(z)} \text { where }|\phi(z)| \leq 1
$$

Taking the logarithmic derivative of this expression we get

$$
\frac{g^{\prime \prime}(z)}{g^{\prime}(z)}=\frac{-2\left(z \phi^{\prime}(z)+\phi(z)\right)}{1-z^{2} \phi^{2}(z)}
$$

Using lemma 2 (ii) and simplifying we get

$$
\left|\frac{g^{\prime \prime}(z)}{g^{\prime}(z)}\right| \leq \frac{2[|z|+|\phi(z)|]}{\left(1-|z|^{2}\right)(1+|z||\phi(z)|)} \leq \frac{2}{1-|z|^{2}} .
$$

Thus

$$
\operatorname{Re}\left\{\frac{z g^{\prime \prime}(z)}{g^{\prime}(z)}+1\right\} \geq 1-\frac{2|z|}{1-|z|^{2}}
$$

Using this estimate in (3.1) we get

$$
\operatorname{Re}\left\{\frac{z f^{\prime \prime}(z)}{f^{\prime}(z)}+1\right\} \geq 1-\frac{2|z|}{1-|z|^{2}}-\frac{|z|}{1-|z|}=\frac{1-3|z|-2|z|^{2}}{1-|z|^{2}}
$$

This last expression is positive for $|z|<(\sqrt{17}-3) / 4$. To show that the estimate obtained is sharp we consider $f(z)$ such that $f^{\prime}(z)=\frac{(1+z)^{2}}{1-z}$ with $g(z)=-z-2 \log (1-z)$. This $f(z)$ satisfies the hypotheses of the theorem. However

$$
\frac{z f^{\prime \prime}(z)}{f^{\prime}(z)}+1=\frac{1+3 z-2 z^{2}}{1-z^{2}}
$$

This last expression vanishes at $z=(3-\sqrt{17}) / 4$.

THEOREM 5. Suppose $f(z)=z+a_{2} z^{2}+\ldots$ is analytic for $|z|<1$ and $g(z)=z+b_{2} z^{2}+\ldots$ is analytic for $|z|<1$ and $\operatorname{Re}\left\{g^{\prime}(z)\right\}>1 / 2$ for $|z|<1$. If $\left|\frac{f^{\prime}(z)}{g^{\prime}(z)}-1\right|<1$ for $|z|<1$, then $f(z)$ maps $|z|<r_{0}$ onto a convex domain, where $r_{0}$ is the smallest positive root of $4-4 r-13 r^{2}-2 r^{3}-r^{4}=0$. The result is sharp.

PROOF. Since $\operatorname{Re}\left\{g^{\prime}(z)\right\}>1 / 2$ for $|z|<1$, we have by lemma 1 with $\alpha=1 / 2$, 
$g^{\prime}(z)=\frac{1}{1+z \phi(z)} \cdot \quad$ Thus

From (3.1) we get

$$
\frac{g^{\prime \prime}(z)}{g^{\prime}(z)}=\frac{z \phi^{\prime}(z)+\phi(z)}{1+z \phi(z)}
$$

$$
\begin{aligned}
& \operatorname{Re}\left\{\frac{z f^{\prime \prime}(z)}{f^{\prime}(z)}+1\right\} \geq \operatorname{Re}\left\{\frac{z g^{\prime \prime}(z)}{g^{\prime}(z)}+1-\frac{|z|}{1-|z|}\right\} \\
& =\operatorname{Re}\left\{\frac{z g^{\prime \prime}(z)}{g^{\prime}(z)}+\frac{1-2|z|}{1-|z|}\right\} \\
& =\operatorname{Re}\left\{\frac{-z^{2} \phi^{\prime}(z)-z \phi(z)}{1+z \phi(z)}+\frac{1-2|z|}{1-|z|}\right\} .
\end{aligned}
$$

Therefore, $\quad \operatorname{Re}\left\{\frac{z f^{\prime \prime}(z)}{f^{\prime}(z)}+1\right\} \quad$ is positive if

$$
\operatorname{Re}\left\{\frac{1-3|z|+|z|(1-z \phi(z))-(1-|z|) z^{2} \phi^{\prime}(z)}{(1-|z|)(1+z \phi(z))}\right\}>0 \text {. }
$$

This will be true if

$$
\begin{gathered}
\operatorname{Re}\left\{\left[|z|(1-z \phi(z))-\left\{(3|z|-1)+(1-|z|) z^{2} \phi^{\prime}(z)\right\}\right][1+z \phi(z)]^{\star}\right\}>0, \\
\text { (asterisks denote the conjugate of a complex number) }
\end{gathered}
$$

$\operatorname{Re}\left\{|z|\left(1-|z|^{2}|\phi(z)|^{2}\right)-\left[(3|z|-1)+(1-|z|) z^{2} \phi^{\prime}(z)\right][1+z \phi(z)]{ }^{*}\right\}>0$, $\operatorname{Re}\left\{\left[(3|z|-1)+(1-|z|) z^{2} \phi^{\prime}(z)\right][1+z \phi(z)]^{*}\right\}<|z|\left(1-|z|^{2}|\phi(z)|^{2}\right)$.

By lemma 2, it is easily seen that this last inequality will be true if

$$
3|z|-1+(1-|z|)|z|^{2}\left(\frac{1-|\phi(z)|^{2}}{1-|z|^{2}}\right)<|z|(1-|z||\phi(z)|) \text {. }
$$

This inequality is equivalent to showing

$$
r+3 r^{2}+r^{2}(1+r) x-r^{2} x^{2}<1,
$$

where $|z|=r(0<r<1)$ and $|\phi(z)|=x \quad(0 \leq x \leq 1)$.

Let $p(x)=r+3 r^{2}+r^{2}(1+r) x-r^{2} x^{2}$.

We see that $p(x)$ attains its maximum value $q(r)$ at $x=\frac{1+r}{2}$, consequently

$$
q(r)=r+3 r^{2}+\frac{r^{2}}{4}(1+r)^{2} \text {. }
$$

Since $r+3 r^{2}+\frac{r^{2}}{4}(1+r)^{2},<1$ holds for all $r<r_{0}$, where $r_{0}$ is the smallest positive root of the equation $r+3 r^{2}+\frac{r^{2}}{4}(1+r)^{2}=1$. 
This simplifies to

$$
4-4 r-13 r^{2}-2 r^{3}-r^{4}=0
$$

To show that the estimate obtained above is sharp, we let $g^{\prime}(z)=\frac{1}{1+z \phi(z)}$, where $\phi(z)=\frac{z+b}{1+b z}, b=\frac{1}{2+r_{0}}$ where $r_{0}$ is the smallest positive root of (3.2); and we select $f(z)$ so that $f^{\prime}(z)=(1-z) g^{\prime}(z)$. Since $|\phi(z)|<1$ for $|z|<1$, we have $\operatorname{Re} g^{\prime}(z)>1 / 2$ for $|z|<1$. Thus $f(z)$ satisfies the hypotheses of the theorem, and

$$
\frac{z f^{\prime \prime}(z)}{f^{\prime}(z)}+1=\frac{1+(2 b-2) z+\left(2 b^{2}-5 b-1\right) z^{2}-4 b^{2} z^{3}-b z^{4}}{(1-z)(1+b x)\left(1+2 b z+z^{2}\right)} \text {. }
$$

Setting $z=r_{0}$ and $b=\frac{1}{2+r_{0}}$, we see that the numerator of the above expression is

$$
\left(1+r_{0}\right)\left(4-4 r_{0}-13 r_{0}^{2}-2 r_{0}^{3}-r_{0}^{4}\right)
$$

which vanishes.

Theorems 4 and 5 give the radius of convexity for the class of functions $f(z)$ associated with $g(z)$ such that $\operatorname{Re} g^{\prime}(z)>\alpha$ when $\alpha=0$ and $1 / 2$. For $\alpha \neq 0,1 / 2$ our method seems to give only estimates for $r_{c}$ the radius of convexity and determination of $r_{c}$ is still open.

\section{REFERENCES}

1. W. K. Hayman, Multivalent Functions, Cambridge University Press, Cambridge, 1958.

2. T. H. MacGregor, A Class of Univalent Functions, Proc. Am. Math. Soc. 15 (1964), 311-317.

3. Z. Nehari, Conforma1 Mapping, McGraw Hill, New York, 1952.

4. J. S. Ratti, The Radius of Convexity of Certain Analytic Functions, J . of Pure and App. Math. 1 (1970), 30-36. 


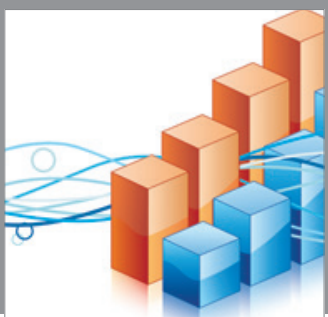

Advances in

Operations Research

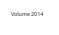

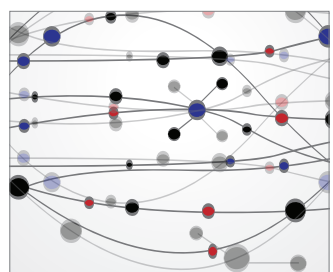

\section{The Scientific} World Journal
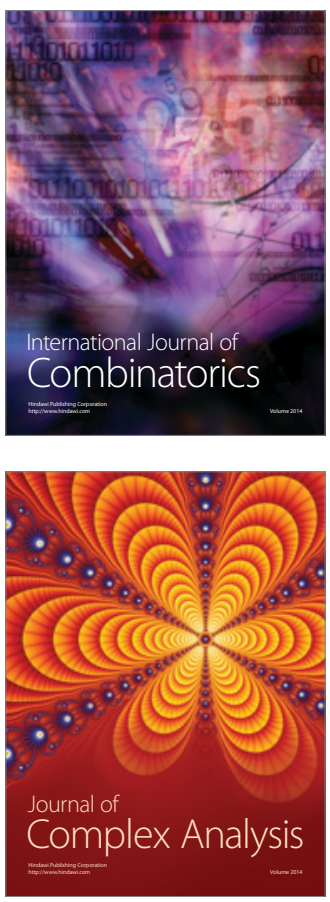

International Journal of

Mathematics and

Mathematical

Sciences
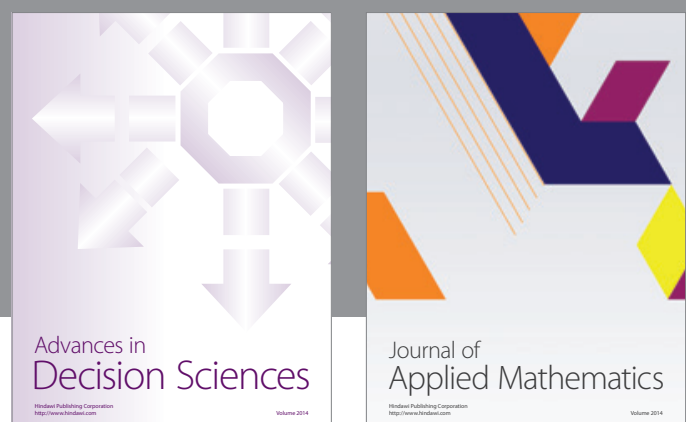

Journal of

Applied Mathematics
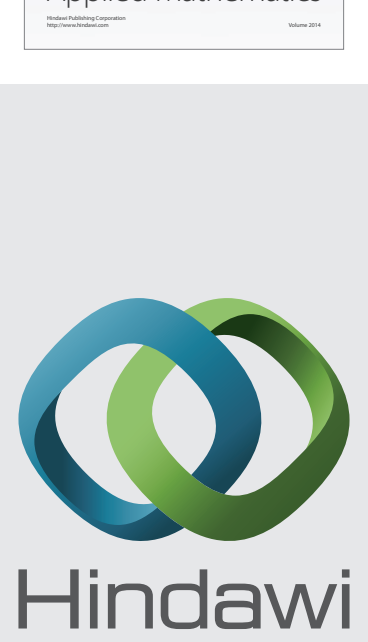

Submit your manuscripts at http://www.hindawi.com
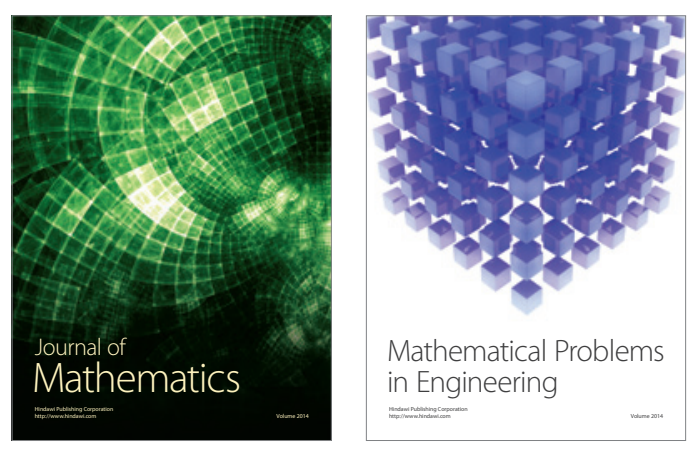

Mathematical Problems in Engineering
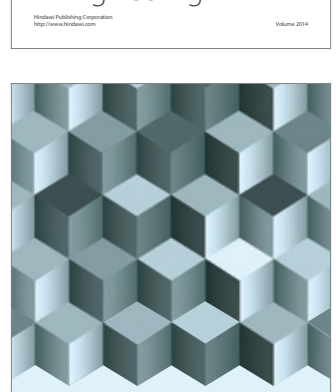

Journal of

Function Spaces
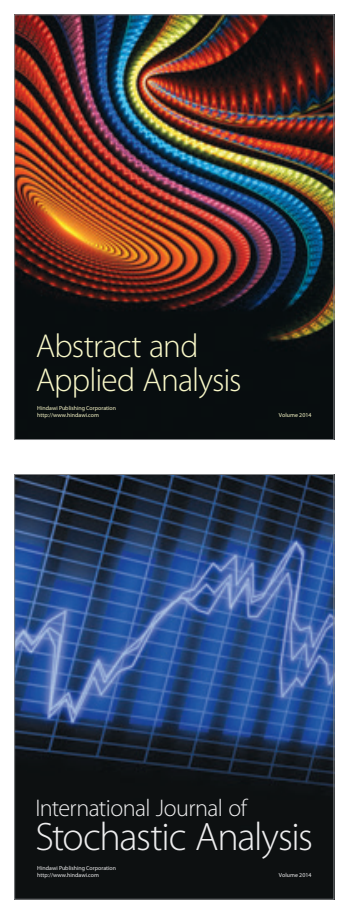

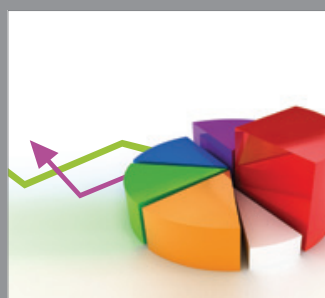

ournal of

Probability and Statistics

Promensencen
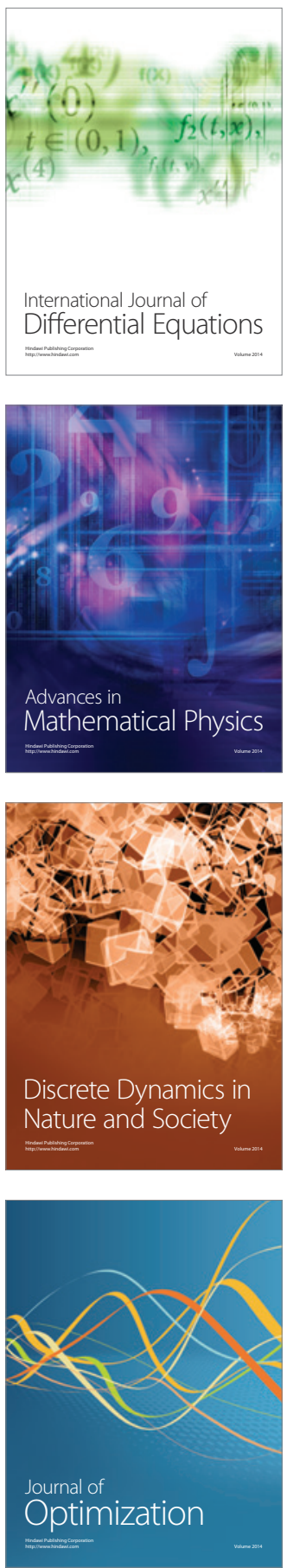